\title{
Triple-layer remote phosphor structure: A potential packaging configuration to enhance both color quality and lumen efficiency of 6,000-8,500 K WLEDs
}

\author{
Phan Xuan Le ${ }^{1}$, Sang Dang $\mathrm{Ho}^{2, *}$, Nguyen Doan Quoc Anh ${ }^{2}$, Hsiao-Yi Lee ${ }^{3}$ \\ ${ }^{1}$ Faculty of Engineering, Van Lang University, 69/68 Dang Thuy Tram Street, Ward 13, Binh Thanh District, Ho Chi Minh \\ City, Vietnam \\ ${ }^{2}$ Faculty of Electrical and Electronics Engineering, Ton Duc Thang University, Ho Chi Minh City, Vietnam \\ ${ }^{3}$ Department of Electrical Engineering, National Kaohsiung University of Science and Technology, Kaohsiung, Taiwan
}

\begin{abstract}
To achieve further enhancement in the lighting quality of white light-emitting diodes (WLEDs), this study proposed a packaging structure with three different phosphor layers, called triple-layer remote phosphor structure. This structure can provide an overall control over the light color distribution of WLEDs. The yellow-green-emitting $\mathrm{CaAl}_{2} \mathrm{O}_{4}: \mathrm{Mn}^{2+}$ phosphor and red-emitting $\mathrm{CaMoO}_{4}: \mathrm{Eu}^{3+}$ phosphor are used along with the original yellow-emitting YAG:Ce ${ }^{3+}$ phosphor to fabricate the triple-layer structure. The concentration of yellow-emitting YAG:Ce ${ }^{3+}$ phosphor is required to be decreased as the concentrations of other phosphors increase to keep the predetermined correlated color temperatures. The color rendering index (CRI) and the color quality scale (CQS) are measured to reach a thorough color quality assessment for WLEDs. The color management can be achieved by adjusting the concentration of red-emitting $\mathrm{CaMoO}_{4}: \mathrm{Eu}^{3+}$ phosphor to enhance the red emission. In addition, adjustment of the concentration of yellow-green-emitting $\mathrm{CaAl}_{2} \mathrm{O}_{4}: \mathrm{Mn}^{2+}$ phosphor can result in higher luminous efficiency owing to its control over the green light components. Higher CRI is observed when $\mathrm{CaMoO}_{4}: \mathrm{Eu}^{3+}$ concentration increases, while an increase in $\mathrm{CaAl}_{2} \mathrm{O}_{4}: \mathrm{Mn}^{2+}$ phosphor leads to much lower CRI. The CQS - on the other hand - is remarkably high when the $\mathrm{CaMoO}_{4}: \mathrm{Eu}^{3+}$ concentration range is about $10 \mathrm{wt} \%-14 \mathrm{wt} \%$, regardless of the proportion of the $\mathrm{CaAl}_{2} \mathrm{O}_{4}: \mathrm{Mn}^{2+}$ phosphor. Furthermore, $40 \%$ enhancement in luminous efficiency is also achieved since light scattering is minimized by the boosted green-light emission spectra. Manufacturers can take these findings as reference to fabricate high-quality WLED lights that fulfill all their requirements.
\end{abstract}

Keywords: $\mathrm{WLEDS}_{\mathrm{C}} \mathrm{CaMoO}_{4}: \mathrm{Eu}^{3+}, \mathrm{CaAl}_{2} \mathrm{O}_{4}: \mathrm{Mn}^{2+}$, luminous efficiency, color quality, remote phosphor structure, triple-layer phosphor structure

\section{Introduction}

Solid-state lighting (SSL) has been widely applied in modern illuminations as it consumes less energy and generates less heat than other traditional lighting sources, such as incandescent bulbs and fluorescent tubes [1]. Therefore, white lightemitting diodes (WLEDs), the main light source of SSL, have received significant recognition due to their ability to offer higher lighting efficiency, lower energy consumption, and lower production cost [2]. WLEDs have also been reported to be used under various environmental conditions due to their robustness and long life span [3]. How-

\footnotetext{
*E-mail: hodangsang@tdtu.edu.vn
}

ever, the luminous efficiency of WLEDs must be further improved for a wide range of applications in SSL. Generally, the lights generated from traditional LEDs are composed of the blue lights from the LED chip and the converted yellow light from the yellow-emitting phosphor layer $[4,5]$. One of the most popular techniques to fabricate a WLED package is freely dispersed phosphor coating. This method utilizes the combination between encapsulated transparent resin and the phosphor powder, which is then dispersed onto the LED chip. Though this method offers a simple fabricating process, affordable production cost, and flexible modification of the thickness of the phosphor layer, the angular color uniformity of the WLED is poor due to 
the inhomogeneity in the distributions of blue and yellow lights $[6,7]$. Thus, it is not suitable for today's high-power WLEDs. The conformal phosphor coating method has been proposed to address the problems of the previous method [8]. Conformal phosphor coating can give uniform light distribution, leading to more homogeneous angular correlated color temperatures (CCTs) [9, 10]. Yet, this method yields low lumen output due to the strong backscattering effect.

Several studies have previously analyzed the remote phosphor package for WLEDs, in which separation between the phosphor film and the LED chip is used to minimize the heating damages to the phosphor materials and to promote better lumen efficiency [11]. Enhanced light extraction by an internal reflection luminaire structure is introduced in this approach, and it is capable of enhancing the light extraction efficiency of WLEDs by at least $26 \%$ [12]. Additionally, another structure with an embedded air gap provides sufficient reflection of downward light to promote better luminescence [13]. These studies also pinpoint the importance of phosphor materials, besides the packaging design, in determining the luminous efficiency of a WLED device. Particularly, once the phosphor concentration increases, the reabsorption loss in the phosphor film becomes significant and, as a result, causes considerable degradation in the emitted luminous flux, which is more noticeable in the low-CCT WLED packages [14-17]. Therefore, it is crucial to obtain higher blue and yellow light emissions while minimizing the loss of backscattered and reflected light to achieve optical improvements in high-quality WLED lamps.

Considering the above-mentioned issues, this research paper presents a triple-layer remote phosphor structure as a more practical solution for higher WLED optical performances. The WLED models used in the experiments have an average CCT range of $6,000 \mathrm{~K}-8,500 \mathrm{~K}$. The phosphors selected for the fabrication of the triple-layer phosphor LED configuration are redemitting $\mathrm{CaMoO}_{4}: \mathrm{Eu}^{3+}$ phosphor, green-emitting $\mathrm{CaAl}_{2} \mathrm{O}_{4}: \mathrm{Mn}^{2+}$ phosphor, and yellow-emitting YAG: $\mathrm{Ce}^{3+}$ phosphor. The $\mathrm{CaAl}_{2} \mathrm{O}_{4}: \mathrm{Mn}^{2+}$ phosphor - with a broad range of green emission spectra
- can help increase the green-light components in the packages and thus is beneficial to the luminous flux. Meanwhile, the red-emitting $\mathrm{CaMoO}_{4}: \mathrm{Eu}^{3+}$ phosphor can improve the color homogeneity with its high red spectral intensity. The results from experiments and calculations in the study point out that the color quality of the LED package is optimized owing to the balanced distribution among the red, green, and yellow colors, while obtaining minimum reduction in lumen output. We believe that this study can contribute greatly to the enhancement of LED optical performances, especially in terms of color uniformity and light extraction efficiency.

\section{Preparation}

\subsection{Preparation of green and red phos- phor materials}

The compositions of green-emitting $\mathrm{CaAl}_{2} \mathrm{O}_{4}: \mathrm{Mn}^{2+}$ phosphor and red-emitting $\mathrm{CaMoO}_{4}: \mathrm{Eu}^{3+}$ phosphor are described in Tables 1 and 2, respectively [18]. The preparation of phosphor material plays an important role in achieving precise experimental results.

Table 1. Composition of yellow-green-emitting $\mathrm{CaAl}_{2} \mathrm{O}_{4}: \mathrm{Mn}^{2+}$ phosphor

\begin{tabular}{lll}
\hline Ingredient & Mole (\%) & By weight (g) \\
\hline \hline $\mathrm{CaCO}_{3}$ & 93 & 93 \\
$\mathrm{Al}_{2} \mathrm{O}_{3}$ & 200 (of $\mathrm{Al})$ & 102 \\
$\mathrm{MnCO}_{3}$ & 2 & 2.3 \\
$\mathrm{CaF}_{2}$ & 5 & 3.9 \\
\hline
\end{tabular}

To fabricate the green-emitting $\mathrm{CaAl}_{2} \mathrm{O}_{4}: \mathrm{Mn}^{2+}$ phosphor, the initial step is to mix the ingredients well in water, let this slurry dry in air, and subsequently powderize it. The next step is to put this powder into a covered alumina crucible, let the flow of $\mathrm{CO}$ into this container, and fire at $1,300{ }^{\circ} \mathrm{C}$ for 1 hour. Once the firing is completed, the product is taken out and powderized. Subsequently, another round of firing is carried out for 1 hour at a temperature of $1,200{ }^{\circ} \mathrm{C}$ in an open quartz boat with $\mathrm{CO}$ flows. The fired powder is washed in a solution of $20 \mathrm{~g} \mathrm{NH}_{2} \mathrm{Cl}$ mixed in $1 \mathrm{~L}$ of water. Finally, it 
is washed again in plain water several times. The $\mathrm{CaAl}_{2} \mathrm{O}_{4}: \mathrm{Mn}^{2+}$ phosphor obtained has a yellowgreen emission color, with emission peak and width of $2.28 \mathrm{eV}$ and $0.27 \mathrm{eV}$, respectively.

Table 2. Composition of red-emitting $\mathrm{CaMoO}_{4}: \mathrm{Eu}^{3+}$ phosphor

\begin{tabular}{lll}
\hline Ingredient & Mole (\%) & By weight (g) \\
\hline \hline $\mathrm{CaCO}_{3}$ & 90 & 90 \\
$\mathrm{Eu}_{2} \mathrm{O}_{3}$ & $5($ of Eu) & 8.8 \\
$\mathrm{NaHCO}_{3}$ & 5 & 4.2 \\
$\mathrm{MoO}_{3}$ & 105 & 151 \\
\hline
\end{tabular}

Similarly, the preparation process of redemitting $\mathrm{CaMoO}_{4}: \mathrm{Eu}^{3+}$ phosphor includes two rounds of firing. First, the ingredients of $\mathrm{CaMoO}_{4}: \mathrm{Eu}^{3+}$ are mixed by dry grinding or milling. Second, the first firing is carried out at $1,000{ }^{\circ} \mathrm{C}$ for $1 \mathrm{~h}$ in an open quartz boat filled with air. Then, the fired mixture is processed into a powder before the second firing is conducted. In the next firing, which also lasts $1 \mathrm{~h}$, the powder is put into the same container but under different conditions: $\mathrm{O}_{2}$ is added into the boat and the temperature is set at $1,100^{\circ} \mathrm{C}$. The product is then washed in $\mathrm{NaOH}$-water or $\mathrm{KOH}$-water solution and washed again with plain water several times, until it becomes neutral. The final product, the $\mathrm{CaMoO}_{4}: \mathrm{Eu}^{3+}$ phosphor particles, emits red lights that peak at 2.02 and $2.03 \mathrm{eV}$, approximately.

\subsection{Simulation of triple-layer remote phosphor configuration}

The WLED simulation is based on the actual WLED model having high thermal stability; the photograph and specifications of this model are displayed in Figure 1A, 1B, respectively. It is essential to have the simulated model similar to the actual one to minimize the influences of other factors, such as wavelength, waveform, intensity of lights, and temperature generated during the operation of LED devices, on the obtained results. The normalized cross correlation of the simulation and actual packages showed $99.6 \%$ similarity. The simulation process was carried out with support from the LightTools 9.0 software using the Monte Carlo method. The simulated LEDs packed with triplelayer remote phosphor structures have CCTs of $6,000 \mathrm{~K}, 7,000 \mathrm{~K}$, and $8,500 \mathrm{~K}$. An illustration of the cross section of the simulated triple-layer phosphor LED package is presented in Figure 1C, while Figure $1 \mathrm{D}$ shows its 3D model.

Specifically, this WLED physical package is mainly composed of a reflector, three phosphor layers, and 9 blue chips. The reflector's bottom, top, and height have dimensions of $8 \mathrm{~mm} \times 9.85 \mathrm{~mm} \times 2.07 \mathrm{~mm}$, respectively. Each phosphor layer has a thickness of $0.08 \mathrm{~mm}$, while each blue chip has a square base of $1.14 \mathrm{~mm}$ and a height of $0.15 \mathrm{~mm}$, with radiant flux emission of $1.16 \mathrm{~W}$ at $455 \mathrm{~nm}$ wavelength. The WLED simulated model with three-layered remote phosphor structure can be described as follows. The blue chips are attached to the reflector's cavity and covered with yellow-emitting phosphor layers. Then, the green-emitting phosphor layer is formed above the yellow layer, and the red-emitting phosphor is put on top, as can be seen in Figure 1C. In Figure $1 \mathrm{E}$, we present the absorption and emission spectra of yellow-emitting YAG: $\mathrm{Ce}^{3+}$ phosphor. Figure $1 \mathrm{~F}$ exhibits the measured spectra of red-emitting $\mathrm{CaMoO}_{4}: \mathrm{Eu}^{3+}$ phosphor including the excitation and emission spectra. The phosphors' concentrations are modified in the range of $2 \mathrm{wt} \%-20 \mathrm{wt} \%$. Meanwhile, the CCTs are stable with control over the concentration of yellow-emitting YAG: $\mathrm{Ce}^{3+}$ phosphor layer.

\section{Computation and discussion}

Figure 2 shows the changes of CRI in connection with the concentration of red-emitting and green-emitting phosphor layers in the range of $2 \mathrm{wt} \%-20 \mathrm{wt} \%$. In particular, when the concentration of red $\mathrm{CaMoO}_{4}: \mathrm{Eu}^{3+}$ layer increases, the CRI also goes up. When $20 \% \mathrm{CaMoO}_{4}: \mathrm{Eu}^{3+}$ is applied, the structure achieves the highest CRI values. Conversely, the green-emitting $\mathrm{CaAl}_{2} \mathrm{O}_{4}: \mathrm{Mn}^{2+}$ phosphor is not beneficial to the color rendering index. As can be seen, the CRI decreases continuously as the concentration of $\mathrm{CaAl}_{2} \mathrm{O}_{4}: \mathrm{Mn}^{2+}$ increases to $20 \mathrm{wt} \%$, regardless of the increase in red-emitting phosphor concentration. Thus, to get 


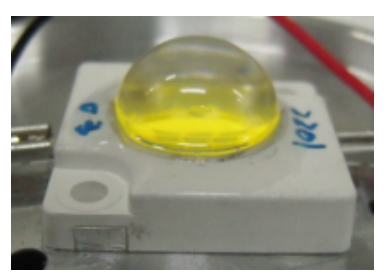

(A)
Lead frame: $4.7 \mathrm{~mm}$ Jentech Size-S

LED chip: $\mathrm{V} 45 \mathrm{H}$

Die attach: Sumitomo 1295SA

Gold Wire: 1.0 mil

Phosphor: ITC NYAG4_EL

(B)

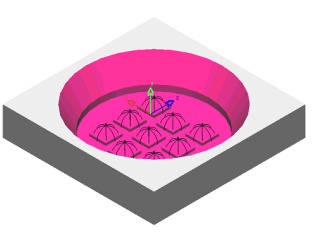

(D)

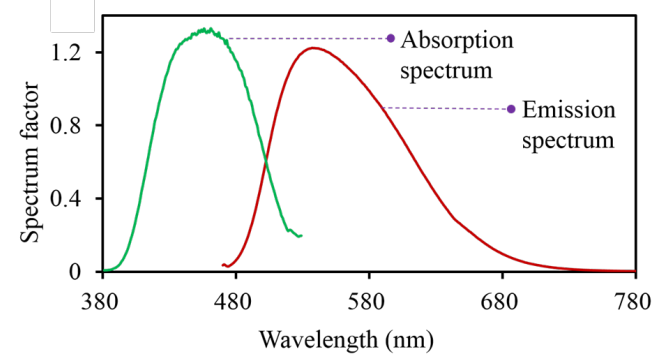

(E)

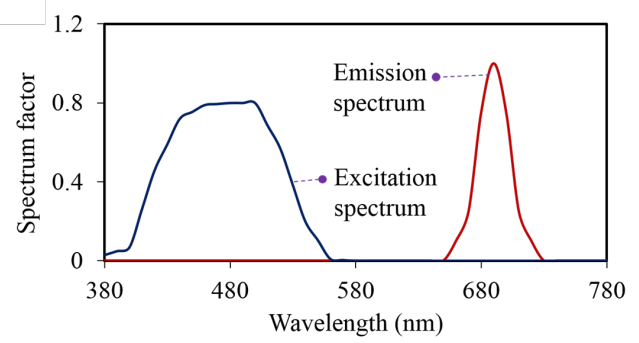

(F)

Fig. 1. (A) WLEDs; (B) its parameters; (C) illustration of triple-layer remote phosphor configuration; (D) the simulation of WLEDs; (E) the measured spectra of the yellow-emitting YAG:Ce ${ }^{3+}$ phosphor; (F) the measured spectra of the red-emitting $\mathrm{CaMoO}_{4}: \mathrm{Eu}^{3+}$ phosphor. WLEDs, white light-emitting diodes

better CRI, a higher red-emitting $\mathrm{CaMoO}_{4}: \mathrm{Eu}^{3+}$ phosphor concentration is required to strengthen the red emission spectrum. However, the greenemitting phosphor concentration must be limited since high concentration of $\mathrm{CaAl}_{2} \mathrm{O}_{4}: \mathrm{Mn}^{2+}$ apparently leads to high proportion of green-light components, which is not favorable for CRI. Besides, the increase in green-emitting phosphor concen- tration degrades the red-emitting phosphor conversion energy, as the lights emitted from the chip reach the green-emitting phosphor layer before being transmitted to the red layer due to the order of the phosphor layers in the triple-layer structure. Consequently, it is essential to limit the concentration of the $\mathrm{CaAl}_{2} \mathrm{O}_{4}: \mathrm{Mn}^{2+}$ phosphor to the lowest to obtain better CRI for the LED package. Though 
WLED devices with high CRI are somehow desirable and sold with high prices in the market, this parameter is not able to evaluate all the aspects of white-light color quality. In fact, CRI is used to determine the ability of a light source to show the true color of the illuminated objects. In addition, two other factors are important in the evaluation of color quality: the preference of viewers and the color coordinates; the parameter that can cover all the three criteria is the color quality scale (CQS) [19]. This implies that CQS is more powerful and difficult to manage than CRI.

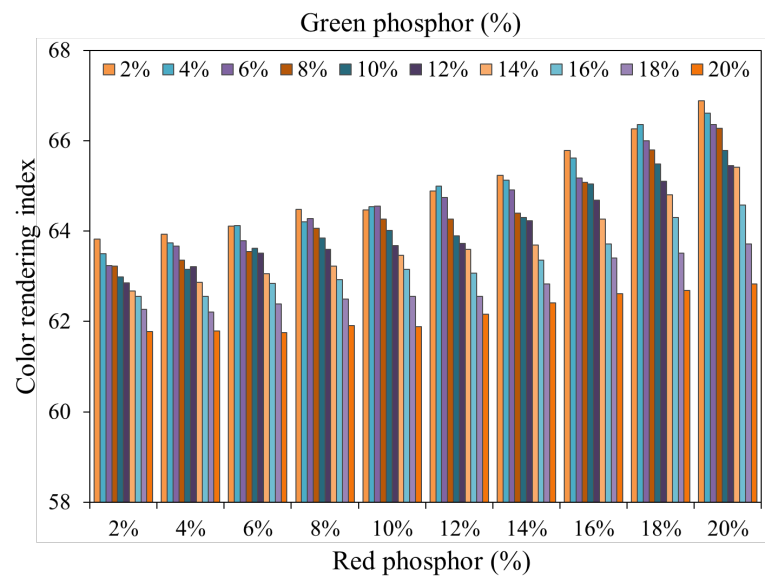

Fig. 2. Color rendering index of triple-layer remote phosphor structure as a function of red-emitting $\mathrm{CaMoO}_{4}: \mathrm{Eu}^{3+}$ and green-emitting $\mathrm{CaAl}_{2} \mathrm{O}_{4}: \mathrm{Mn}^{2+}$ phosphors

Figure 3 displays the CQS of the triple-layer WLED package with different concentrations of red-emitting $\mathrm{CaMoO}_{4}: \mathrm{Eu}^{3+}$ phosphor and greenemitting $\mathrm{CaAl}_{2} \mathrm{O}_{4}: \mathrm{Mn}^{2+}$ phosphor layers. The point of this experiment is to figure out whether it is possible to achieve high CQS by adjusting the phosphor concentration. Similar to the CRI, redemitting $\mathrm{CaMoO}_{4}: \mathrm{Eu}^{3+}$ phosphor can improve the CQS when its concentration increases. Moreover, as can be seen in Figure 3, the modification of proportion of green-emitting $\mathrm{CaAl}_{2} \mathrm{O}_{4}: \mathrm{Mn}^{2+}$ phosphor also benefits the CQS. Particularly, considering the CQS values at a certain red-emitting phosphor concentration, the difference between the CQS values on changing the green-emitting phosphor concentration is insignificant. This proves that
$\mathrm{CaAl}_{2} \mathrm{O}_{4}: \mathrm{Mn}^{2+}$ and $\mathrm{CaMoO}_{4}: \mathrm{Eu}^{3+}$ are appropriate to enhance the CQS of the generated white lights. The triple-layer packaging structure can heighten the CQS for WLED devices because it has the ability to balance the yellow, green, and red light colors, the three crucial colors of white-light formation. Specifically, the yellow YAG:Ce ${ }^{3+}$ concentration decreases to keep the stability of color temperature since the concentration of the red $\mathrm{CaMoO}_{4}: \mathrm{Eu}^{3+}$ or green $\mathrm{CaAl}_{2} \mathrm{O}_{4}: \mathrm{Mn}^{2+}$ increases.

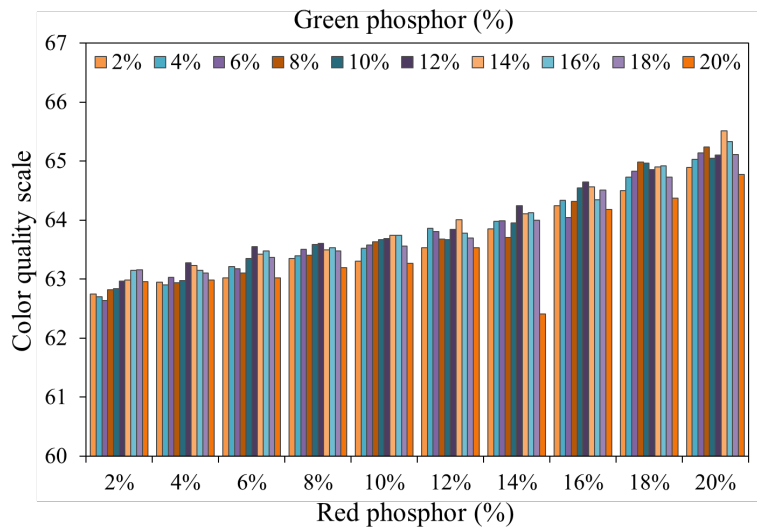

Fig. 3. CQS of triple-layer remote phosphor structure as a function of red-emitting $\mathrm{CaMoO}_{4}: \mathrm{Eu}^{3+}$ and green-emitting $\mathrm{CaAl}_{2} \mathrm{O}_{4}: \mathrm{Mn}^{2+}$ phosphors. CQS, color quality scale

The color balance is critical to the performance of CQS, as proven above. Thus, to maintain high CQS, it is essential to control the proportion of green-emitting $\mathrm{CaAl}_{2} \mathrm{O}_{4}: \mathrm{Mn}^{2+}$ phosphor to attain sufficient amount of green light color for the best color balance among the green, red, and yellow components. From Figure 3, we can observe the increase in CQS with the greenemitting $\mathrm{CaAl}_{2} \mathrm{O}_{4}: \mathrm{Mn}^{2+}$ phosphor in the $2 \mathrm{wt} \%-$ $10 \mathrm{wt} \%$ concentration range. Moreover, the CQS reaches the highest values when $\mathrm{CaAl}_{2} \mathrm{O}_{4}: \mathrm{Mn}^{2+}$ is from $10 \mathrm{wt} \%$ to $14 \mathrm{wt} \%$, since the abundant yellow-light amounts are reduced. In other words, at $10 \mathrm{wt} \%-14 \mathrm{wt} \%$ of $\mathrm{CaAl}_{2} \mathrm{O}_{4}: \mathrm{Mn}^{2+}$, the proportion of green light is sufficient to maintain a balance among the three colors, and the CQS is enhanced consequently. When the concentration of $\mathrm{CaAl}_{2} \mathrm{O}_{4}: \mathrm{Mn}^{2+}$ is $>14 \mathrm{wt} \%$, the CQS starts to de- 
cline due to the excessive green-light proportion, damaging the color balance.

In fact, compared to the conformal phosphor coating or in-cup phosphor packaging structure, accomplishing improvement in color uniformity of remote phosphor structure is more difficult and complex, especially the one with high CCT ranging from $7,000 \mathrm{~K}$ to $8,500 \mathrm{~K}$. The use of triplelayer remote phosphor structure aims to address this problem since the results indicate that better CQS is observed in not only low CCT-WLED but also high CCT-WLED packages. Besides having a balanced distribution among the color elements, as mentioned herein, the triple-layer phosphor structure also boosts light scattering in the LED package, leading to better light mixing and thus enhancement of the white-light quality. However, the problem is that when the scattering is better, the lumen output tends to be lower. Thus, the analysis of lumen output in connection with the increase in light scattering is essential.

To carry out the analysis on the WLED efficiency with triple-layer remote phosphor structure, calculation of its transmitted blue light and converted yellow light is carried out. The computation is based on the Mie scattering theory and starts with the expressions of dual-layer remote phosphor structure to demonstrate the better efficiency of the triple-layer structure.

For the dual-layer structure, its transmittedblue-light and converted-yellow-light computation is presented as follows. The thickness of each phosphor layer in this package is set as $h[19,20]$.

$$
\begin{aligned}
& P B_{2}=P B_{0} e^{-\alpha_{B_{2}} h} e^{-\alpha_{B_{2}} h}=P B_{0} e^{-2 \alpha_{B_{2}} h} \\
P Y_{2}= & \frac{1}{2} \frac{\beta_{2} P B_{0}}{\alpha_{B_{2}}-\alpha_{Y_{2}}}\left[e^{-\alpha_{Y_{2}} h}-e^{-\alpha_{B_{2}} h} e^{-\alpha_{Y_{2}} h}\right] \\
+ & \frac{1}{2} \frac{\beta_{2} P B_{0}}{\alpha_{B_{2}}-\alpha_{Y_{2}}}\left[e^{-\alpha_{Y_{2}} h}-e^{-\alpha_{B_{2}} h}-e^{-\alpha_{B_{2}} h}\right] e^{-\alpha_{Y_{2}} h} \\
+ & \frac{1}{2} \frac{\beta_{2} P B_{0}}{\alpha_{B_{2}}-\alpha_{Y_{2}}}\left[e^{-\alpha_{Y_{2}} h}-e^{-\alpha_{B_{2}} h}\right] \\
= & \frac{1}{2} \frac{\beta_{2} P B_{0}}{\alpha_{B_{2}}-\alpha_{Y_{2}}}\left[e^{-2 \alpha_{Y_{1}} h}-e^{-2 \alpha_{B_{1}} h}\right]
\end{aligned}
$$

Next, in the triple-layer structure, the thickness of each phosphor film is $\frac{2 \mathrm{~h}}{3}$; therefore, the calculation of its transmitted blue light and converted yellow light can be expressed as follows:

$$
\begin{aligned}
& P B_{3}=P B_{0} e^{-\alpha_{B_{2}} \frac{2 h}{3}} e^{-\alpha_{B_{2}} \frac{2 h}{3}} e^{-\alpha_{B_{2}} \frac{2 h}{3}} \\
& =P B_{0} e^{-2 \alpha_{B_{3}} h} \\
& P Y_{3}^{\prime}=\frac{1}{2} \frac{\beta_{3} P B_{0}}{\alpha_{B_{3}}-\alpha_{Y_{3}}}\left[e^{-\alpha_{Y_{3}} \frac{2 h}{3}}-e^{-\alpha_{B_{3}} \frac{2 h}{3}}\right] e^{-\alpha_{Y_{3}} \frac{2 h}{3}} \\
& +\frac{1}{2} \frac{\beta_{3} P B_{0} e^{-\alpha_{B_{3}} \frac{2 h}{3}}}{\alpha_{B_{3}}-\alpha_{Y_{3}}}\left[e^{-\alpha_{Y_{3}} \frac{2 h}{3}}-e^{-\alpha_{B_{3}} \frac{2 h}{3}}\right] \\
& =\frac{1}{2} \frac{\beta_{3} P B_{0}}{\alpha_{B_{3}}-\alpha_{Y_{3}}}\left[e^{-\alpha_{Y_{3}} \frac{4 h}{3}}-e^{-2 \alpha_{B_{3}} \frac{4 h}{3}}\right] \\
& P Y_{3}=P Y^{\prime}{ }_{3} e^{-\alpha_{Y_{3}} \frac{2 h}{3}}+P B_{0} \\
& e^{-2 \alpha_{B_{3}} \frac{4 h}{3}} \frac{1}{2} \frac{\beta_{3}}{\alpha_{B_{3}}-\alpha_{Y_{3}}}\left[e^{-\alpha_{Y_{3}} \frac{2 h}{3}}-e^{-\alpha_{B_{3}} \frac{2 h}{3}}\right] \\
& =\frac{1}{2} \frac{\beta_{3} P B_{0}}{\alpha_{B_{3}}-\alpha_{Y_{3}}}\left[e^{-\alpha_{Y_{3}} \frac{4 h}{3}}-e^{-\alpha_{B_{3}} \frac{4 h}{3}}\right] e^{-\alpha_{Y_{3}} \frac{2 h}{3}} \\
& +\frac{1}{2} \frac{\beta_{3} P B_{0} e^{-\alpha_{B_{3}} \frac{4 h}{3}}}{\alpha_{B_{3}}-\alpha_{Y_{3}}}\left[e^{-\alpha_{Y_{3}} \frac{2 h}{3}}-e^{-\alpha_{B_{3}} \frac{2 h}{3}}\right] \\
& =\frac{1}{2} \frac{\beta_{3} P B_{0}}{\alpha_{B_{3}}-\alpha_{Y_{3}}}\left[e^{-\alpha_{Y_{3}} h}-e^{-2 \alpha_{B_{3}} h}\right]
\end{aligned}
$$

Here, in Eqs (1-5), $h$ indicates the phosphor layer's thickness. The triple layer and dual layer are described by the subscripts " 3 " and " 2 ", respectively; $\beta$ is the conversion coefficient when blue light is converted to yellow light in the package, while $\gamma$ indicates the yellow-light reflection coefficient. $P B$ is the blue light intensity and $P Y$ is the intensity of yellow light, both of which combine to give $P B_{0}-$ the light intensity from the LED chip. Besides, $P_{3}$ represents the yellow light transmitted through the two red-emitting and green-emitting phosphor layers; $\alpha_{B}$ and $\alpha_{Y}$ describe the fractions of blue-light and yellow-light energy loss, respectively [21, 22].

Higher lighting performance of the triple-layer structure can be observed, compared to that of the dual-layer structure, as demonstrated in the following expression:

$$
\begin{aligned}
& \frac{\left(P B_{3}-P Y_{3}\right)-\left(P B_{2}+P Y_{2}\right)}{\left(P B_{2}+P Y_{2}\right)} \\
& >\frac{e^{-2 \alpha_{B_{3}} h}-e^{-2 \alpha_{B_{2}} h}}{e^{-2 \alpha_{Y_{3}} h}-e^{-2 \alpha_{B_{2}} h}}
\end{aligned}
$$


The scattering property of the utilized phosphor materials and the scattering cross section $C_{s c a}$ for the phosphor spheres are analyzed and calculated using Mie scattering theory [23, 24]. The BeerLambert law is applied to compute the transmitted light power $[25,26]$ :

$$
I=I_{0} \exp \left(-\mu_{e x t} L\right)
$$

where $I_{0}, L$, and $\mu_{\text {ext }}$ are the incident light power, the thickness of the phosphor layers, and the extinction coefficient, respectively. Here, the extinction coefficient can be calculated using the equation $\mu_{e x t}=N_{r} \cdot C_{e x t}$, in which $N_{r}$ expresses the number density distribution of the particles (in particles per cubic millimeter), and $C_{\text {ext }}$ (in square millimeters) shows the spherical phosphor's extinction cross section.

Expression (6) implies that more phosphor layers will result in higher lumen output for WLED packages. Enhancement in lumen efficiency means that the concentrations of the red-emitting and green-emitting phosphor layers increase. As mentioned earlier, the reduction in yellow-emitting phosphor concentration, as a function of the higher concentration of green-emitting and red-emitting phosphors, is necessary for CCT stability. The decline in yellow-emitting phosphor concentration minimizes the energy loss of lights by scattering to promote luminous intensity. Based on Beer's law and Eq. (7), it can be demonstrated that the higher the light transmission energy, the more is the reduction in the yellow-emitting phosphor concentration, and consequently, the luminous output is intensified. Thus, the increase in either green-emitting $\mathrm{CaAl}_{2} \mathrm{O}_{4}: \mathrm{Mn}^{2+}$ or redemitting $\mathrm{CaMoO}_{4}: \mathrm{Eu}^{3+}$ phosphor film is beneficial to WLED lumen efficiency. However, continuous increase in the concentration of red-emitting or green-emitting phosphor probably ruins the color balance as there are excessive amounts of red or green lights; thus, the considerable degradation in CQS is unavoidable.

Figure 4 demonstrates the increase in luminous flux values following the increase of green-emitting $\mathrm{CaAl}_{2} \mathrm{O}_{4}: \mathrm{Mn}^{2+}$ phosphor concentration. Specifically, the luminous output can be enhanced by $40 \%$

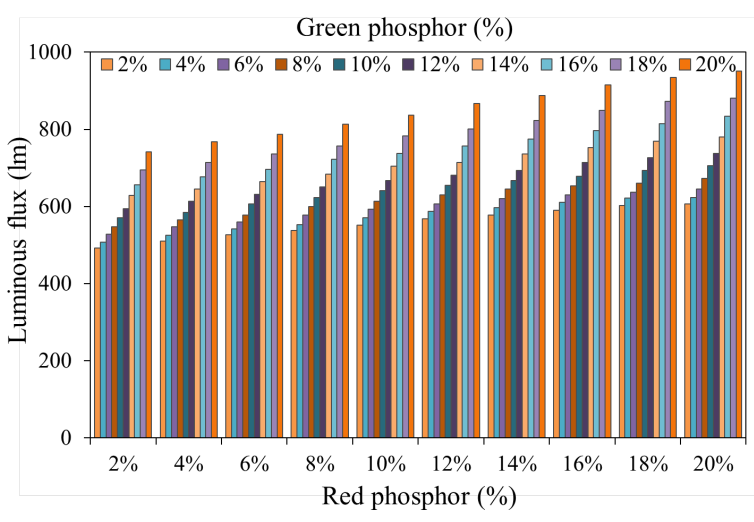

Fig. 4. Lumen output of triple-layer remote phosphor structure as a function of redemitting $\mathrm{CaMoO}_{4}: \mathrm{Eu}^{3+}$ and green-emitting $\mathrm{CaAl}_{2} \mathrm{O}_{4}: \mathrm{Mn}^{2+}$ phosphors

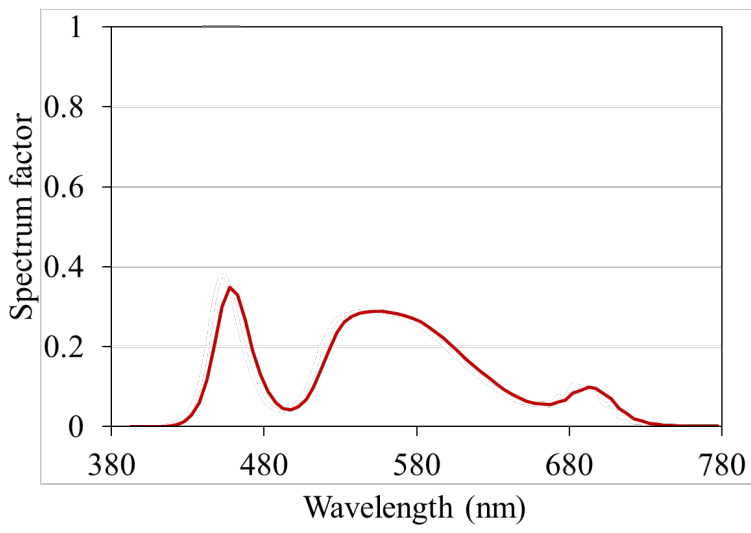

Fig. 5. The emission spectra of WLEDs under varying conditions of the triple-layer structure. WLEDs, white light-emitting diodes

as the green light increases in its proportion and its scattering events, regardless of the changes in red-emitting $\mathrm{CaMoO}_{4}: \mathrm{Eu}^{3+}$ phosphor concentration. Moreover, Figure 5 demonstrates the increase in blue, green, and red spectral intensities. This indicates that the blue light transmission and conversion are better, and the color distribution is enhanced, leading to boosted color uniformity for the WLED package. This means that it is possible to achieve improvement in both CQS and luminous efficiency at the same time using the triple-layer structure. However, it is inadvisable to increase the green-emitting phosphor concentration contin- 
uously, as the color quality deteriorates sharply. According to the experimental results of CQS investigation, the suitable amount of $\mathrm{CaAl}_{2} \mathrm{O}_{4}: \mathrm{Mn}^{2+}$ phosphors is in the range of $10 \mathrm{wt} \%-14 \mathrm{wt} \%$, while the red-emitting $\mathrm{CaMoO}_{4}: \mathrm{Eu}^{3+}$ phosphor can be from $2 \mathrm{wt} \%$ to $20 \mathrm{wt} \%$.

\section{Conclusions}

The application and investigation of the influences of the triple-layer remote phosphor packaging structure for WLEDs are demonstrated in this article. In addition to the yellow-emitting YAG: $\mathrm{Ce}^{3+}$ phosphor, the other two materials used to fabricate the triple-layer structure are red-emitting $\mathrm{CaMoO}_{4}: \mathrm{Eu}^{3+}$ and green-emitting $\mathrm{CaAl}_{2} \mathrm{O}_{4}: \mathrm{Mn}^{2+}$ phosphors. The experimental and analyzed results showed that the triple-layer remote phosphor is suitable and has potential for the enhancement of LED optical performances, especially the color quality and lumen efficiency. In particular, with appropriate concentrations of redemitting and green-emitting phosphors, it is possible to attain higher color uniformity while stabilizing the luminous intensity at high values. The research showed that the concentration of the greenemitting $\mathrm{CaAl}_{2} \mathrm{O}_{4}: \mathrm{Mn}^{2+}$ phosphor should remain between $10 \mathrm{wt} \%$ and $14 \mathrm{wt} \%$, and the red-emitting phosphor concentration may be adjusted from $2 \%$ to $20 \%$. Hence, this study can be a useful reference for manufacturers to obtain a color balance among the green, red, and yellow components of white lights. In other words, application of the triplelayer remote phosphor structure can be considered in WLED manufacturing to accomplish high-colorquality WLEDs with better luminous efficiency.

\section{Acknowledgements}

This research is funded by Van Lang University, website: https : //www . vanlanguni.edu.vn.

\section{References}

[1] Luo G-F, Loan NTP, Tho LV, Anh NDQ, Lee $\mathrm{H}-\mathrm{Y}$. Enhancement of color quality and luminous flux for remote-phosphor LEDs with red-emitting $\mathrm{CaMgSi}_{2} \mathrm{O}_{6}: \mathrm{Eu}^{2+}, \mathrm{Mn}^{2+}$. Mater Sci Pol. 2020;38:40915, doi:10.2478/msp-2020-0045.
[2] Anh NDQ, Ngoc HV. Building superior lighting properties for WLEDs utilizing two-layered remote phosphor configurations. Mater Sci Pol. 2020;38:493-501, doi:10.2478/msp-2020-0044.

[3] Anh NDQ, Vinh NH, Lee H-Y. Effect of redemitting $\mathrm{Sr}_{2.41} \mathrm{~F}_{2.59} \mathrm{~B}_{20.03} \mathrm{O}_{74.8}: \mathrm{Eu}_{0.12}, \mathrm{Sm}_{0.048}$ phosphor on color rendering index and luminous efficacy of white LEDs. Curr Opt Photon. 2017;1:118-24, doi:10.3807/COPP.2017.1.2.118

[4] Anh NDQ, Vinh NH, Lee H-Y, Lee H-Y. Gaussian decomposition method in designing a freeform lens for an LED fishing/working lamp. Curr Opt Photon. 2017;1:233-8, doi:10.3807/COPP.2017.1.3.233.

[5] Sun W-S, Tien C-L, Pan J-W, Yang T-H, Tsuei C-H, Huang Y-H. Simulation and comparison of the lighting efficiency for household illumination with LEDs and fluorescent lamps. J Opt Soc Korea. 2013;17:376-83, doi:10.3807/JOSK.2013.17.5.376.

[6] Hayashida T, Iwasaki H, Masaoka K, Shimizu M, Yamashita T, Iwai W. Appropriate indices for color rendition and their recommended values for UHDTV production using white LED lighting. Opt Express. 2017;25:15010-27, doi: 10.1364/OE.25.015010.

[7] Jiang P-Q, Peng Y, Mou Y, Cheng H, Chen MX, Liu S. Thermally stable multi-color phosphor-inglass bonded on flip-chip UV-LEDs for chromaticitytunable WLEDs. Appl Opt. 2017;56:7921-6, doi: 10.1364/AO.56.007921

[8] Yang Z-F, Xu D-H, Sun J-Y. Synthesis and luminescence properties of $\mathrm{Ba}_{3} \mathrm{Lu}\left(\mathrm{PO}_{4}\right)_{3}: \mathrm{Sm}^{3+}$ phosphor for white light-emitting diodes. Opt Express. 2017;25:A391-401, doi:10.1364/OE.25.00A391.

[9] Lyapin AA, Gushchin SV, Ermakov AS, Kuznetsov SV, Ryabochkina PA, Yu V, et al.. Mechanisms and absolute quantum yield of upconversion luminescence of fluoride phosphors. Chin Opt Lett. 2018;16:091901, doi:10.3788/COL201816.091901.

[10] Jeon S-W, Kim S-H, Choi J, Jang I, Song Y-H, Kim W$\mathrm{H}$, et al. Optical design of dental light using a remote phosphor light-emitting diode package for improving illumination uniformity. Appl Opt. 2018;57:5998-6003, doi: 10.1364/AO.57.005998.

[11] Lee H-S, Kim S-H, Heo J, Chung WJ. Phosphorin-glass with Nd-doped glass for a white LED with a wide color gamut. Opt Lett. 2018;43:627-30, doi:10.1364/OL.43.000627.

[12] Ojo AO, Fond B, Abram C, Wachem BGMV, Heyes AL, Beyrau F. Thermographic laser Doppler velocimetry using the phase-shifted luminescence of BAM:Eu ${ }^{2+}$ phosphor particles for thermometry. Opt Express 2017;25:11833-43, doi:10.1364/OE.25.011833.

[13] Liu K-K, Shan C-X, Zhou R, Zhao Q, Shen D-Z. Largescale synthesis of $\mathrm{ZnO}$ nanoparticles and their application as phosphors in light-emitting devices. Opt Mater Express. 2017;7:2682-90, doi:10.1364/OME.7.002682.

[14] Tang L, Ye HQ, Xiao D. Photo-induced luminescence degradation in $\mathrm{Ce}, \mathrm{Yb}$ co-doped yttrium aluminum 
garnet phosphors. Appl Opt. 2018;57:7627-33, doi: 10.1364/AO.57.007627.

[15] Huang Z, Nie ZQ, Xie MB, Wang YX, Li DY. Excellent optical thermometry based on upconversion emission in $\mathrm{SrMoO}_{4}: \mathrm{Er}^{3+}$ phosphor. Opt Mater Express. 2017;7:2404-10, doi:10.1364/OME.7.002404.

[16] Qi YH, Zhao L, Bian WJ, Yu X, Xu XH, Qiu JB. Energy transfer between $\mathrm{Ce}^{3+}$ and $\mathrm{Sm}^{3+}$ in $\mathrm{Zn}_{2} \mathrm{GeO}_{4}$ phosphor with the native defects for lightemitting diodes. Chin Opt Lett. 2017;15:081601, doi:10.3788/COL201715.081601.

[17] Chen GB, Zhang J. Investigation on optical temperature sensing behaviour for $\mathrm{Y}_{4.67} \mathrm{Si}_{3} \mathrm{O}_{13}: \mathrm{Tm}^{3+}, \mathrm{Yb}^{3+}$ phosphors based on upconversion luminescence. Opt Mater Express. 2018;8:1841-9, doi:10.1364/OME.8.001841.

[18] Yen WM, Weber MJ. Inorganic phosphors: compositions, preparation and optical properties. Boca Raton, FL: CRC Press; 2004, doi:10.1201/9780203506325.

[19] Liu S, Luo XB. LED packaging for lighting applications: design, manufacturing and testing, Chapter 3. Beijing: Chemical Industry Press and John Wiley \& Sons; 2011, doi:10.1002/9780470827857.

[20] Shuai Y, Tran NT, You JP, Shi FG. Phosphor size dependence of lumen efficiency and spatial CCT uniformity for typical white LED emitters. IEEE ECTC, 2025 - 2028; 2012, doi: 10.1109/ECTC.2012.6249118.

[21] Davis W, Ohno Y. Color quality scale. Opt Eng. 2010;49:033602-033602-16, doi:10.1117/1.3360335.
[22] Li B-C, Zhang D-W, Huang Y-S, Ni ZJ, Zhuang S-L. A new structure of multi-layer phosphor package of white LED with high efficiency. Chin Opt Lett. 2010;8:221-3, doi:10.3788/COL20100802.0221.

[23] Liu Z-Y, Liu S, Wang K, Luo X-B. Measurement and numerical studies of optical properties of YAG:Ce phosphor for white light-emitting diode packaging. Appl Opt. 2010;49:247-57, doi:10.1364/AO.49.000247.

[24] Nguyen TMH, Ton TP, Anh NDQ. Eu-activated strontium-barium silicate: a positive solution for improving luminous efficacy and color uniformity of white light-emitting diodes. Mater Sci Pol. 2021;38:594-600, doi:10.2478/msp-2020-0069.

[25] Ton TP, Phuong LNT, Le VT, Anh NDQ, Yuan LH, Luo G-F, et al. Enhancing color quality of WLEDs with dual-layer remote phosphor geometry. Mater Sci Pol. 2021;38:667-74, doi:10.2478/msp-2020-0070.

[26] Liu R, Liu L-J, Liang Y-J. Energy transfer and colortunable luminescence properties of $\mathrm{YVO}_{4}: \mathrm{RE}(\mathrm{RE}=$ $\mathrm{Eu}^{3+}, \mathrm{Sm}^{3+}, \mathrm{Dy}^{3+}, \mathrm{Tm}^{3+}$ ) phosphors via molten salt synthesis. Opt Mater Express. 2018;8:1686-94, doi:10.1364/OME.8.001686. 